\title{
Short-range correlation in the uniform electron gas: Extended Overhauser model
}

\author{
Paola Gori-Giorgi ${ }^{1,2 *}$ and John P. Perdew ${ }^{1}$ \\ ${ }^{1}$ Department of Physics and Quantum Theory Group, \\ Tulane University, New Orleans, Louisiana 70118 USA \\ ${ }^{2}$ Unità INFM di Roma "La Sapienza", Piazzale A. Moro 2, 00185 Rome, Italy
}

(Dated: October 22, 2018)

\begin{abstract}
We use the two-electron wavefunctions (geminals) and the simple screened Coulomb potential proposed by Overhauser [Can. J. Phys. 73, 683 (1995)] to compute the pair-distribution function $g(r)$ for a uniform electron gas, finding the exact $g(0)$ for this model and extending the results from $g(0)$ to $g(r)$. We find that the short-range $\left(r<r_{s}\right)$ part of this $g(r)$ is in excellent agreement with Quantum Monte Carlo simulations for a wide range of electron densities. We are thus able to estimate the value of the second-order $\left(r^{2}\right)$ coefficient of the small interelectronic-distance expansion of the pair-distribution function. The coefficients of the small- $r$ expansion of the spin-resolved $g_{\sigma \sigma^{\prime}}(r)$ have density or $r_{s}$ dependences which we parametrize in a way that makes it easy to find their coupling-constant averages. Their spin-polarization or $\zeta$ dependences are estimated from a proposed spin scaling relation.
\end{abstract}

\section{INTRODUCTION}

The pair-distribution function $g(r)$ for the uniform electron gas is related to the probability of finding a pair of electrons at a distance $r$ from each other. (The expected number of electrons in a shell of volume $4 \pi r^{2} d r$ when another electron is at the origin is given by $n g(r) 4 \pi r^{2} d r$, where $n=N / V$ is the electron density.) In recent vears much attention has been devoted to this quantity, 1 . 3.4 , 5 . 0 mainly because of its relevance in densityfunctional theory: $g(r)$ is the basis of many non-local 19 and semi-local 10 exchange-correlation energy density functionals. A good $g(r)$ for the uniform electron gas is also the starting point for building up the systemaveraged exchange-correlation hole of a many-electron system of non-uniform density.11

After oscillations are averaged out, the pong-range part $(r \rightarrow \infty)$ of $g(r)$ is exactly described 312 by the random-phase approximation (RPA). At intermediate interelectronic distances, $0.5 \lesssim r / r_{s} \lesssim 5$ (where $r_{s}$ is the density parameter, $\left.r_{s}=(4 \pi n / \widetilde{3})^{-1 / 3}\right)$, very reliable information is avajable from Quantum Monte Carlo (QMC) simulations.13.14.15.16 Little is known about the quantitative behavior of $g(r)$ for $r / r_{s} \lesssim 0.5$, except in the high-density limit, 40 and except some estimates of $g(0)$, the value of the pair-distribution function at zero-

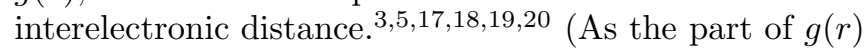
that is most transferable from uniform to non-uniform densities, $g(0)$ plays a special role in spin-density functional theory.19)

In particular, Overhauser 18 presented a simple model for estimating the value of $g(0)$. The model takes into account two-particle correlations by means of a simple screened Coulomb potential with no empirical parameter. In his work Overhauser finds an approximate solution

\footnotetext{
* present address: Dipartimento di Fisica, Università di Roma "La Sapienza", Piazzale A. Moro 2, 00185 Rome, Italy
}

for the dependence of $g(0)$ on the electron density. His result looks realistic and performs surprisingly well in the known high-density limit.

Motivated by this result, in the present work we solve the Overhauser model exactly, i.e., we compute the corresponding pair-distribution function for the uniform electron gas. Comparison of our results with recent QMC simulations 16 shows very good agreement in the range $0.5 \lesssim r / r_{s} \lesssim 1$. We thus have a strong indication that the Overhauser model potential gives accurate quantitative results also in the "unknown" shortest-range region $0 \leq r / r_{s} \lesssim 0.5$. In this way we are able to present a quantitative, reliable estimate for the $r_{s}$ dependence of the $r^{2}$ coefficient of the small- $r$ expansion of $g(r)$. This coefficient is important for new energy density functionals which include the gradient correction to the $r^{2}$ coefficient of the exchange-correlation hole, while its spin resolution (i.e., its $\uparrow \uparrow$ and $\uparrow \downarrow$ contributions, also available in the present treatment) is of interest for functionals based on the Fermi hole curvature.21 Other possible applications are discussed at the end of this article.

The Overhauser model is fully quantum mechanical, and incorporates the effect of exchange on the pairdistribution function in the zero-temperature electron gas. An interesting alternative approach 22 mimics these effects by using a classical pair-distribution function with a non-zero effective temperature.

\section{UNIFORM ELECTRON GAS FROM TWO-PARTICLE WAVEFUNCTIONS}

Following Overhauser, 18 we will construct the pair distribution function from two-electron wavefunctions. The rigorous analogs of these wavefunctions are perhaps the "natural gemipals" that diagonalize the two-electron density matrix;234 the diagonal of this matrix is the pair

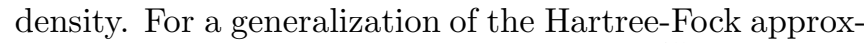
imation from orbitals to geminals, see Ref.25.

If we select a pair of electrons at random in the spin- 
unpolarized uniform gas, there is one chance in four that they will be in the singlet state, $\uparrow \downarrow-\downarrow \uparrow$, and three chances in four that they will be in one of the triplet states, $\uparrow \uparrow, \downarrow \downarrow, \uparrow \downarrow+\downarrow \uparrow$. In the case of no electron-electron interaction, the corresponding two-electron spatial wavefunctions will be

$$
\Psi(\mathbf{r}, \mathbf{R})=\frac{1}{\sqrt{2}} \mathrm{e}^{i \mathbf{K} \cdot \mathbf{R}}\left(\mathrm{e}^{i \mathbf{k} \cdot \mathbf{r}} \pm \mathrm{e}^{-i \mathbf{k} \cdot \mathbf{r}}\right),
$$

where "+" is for the singlet state and "-" is for the triplet state, and

$$
\begin{gathered}
\mathbf{R}=\frac{1}{2}\left(\mathbf{r}_{1}+\mathbf{r}_{2}\right), \quad \mathbf{r}=\mathbf{r}_{2}-\mathbf{r}_{1}, \\
\mathbf{K}=\mathbf{k}_{1}+\mathbf{k}_{2}, \quad \mathbf{k}=\frac{1}{2}\left(\mathbf{k}_{2}-\mathbf{k}_{1}\right) .
\end{gathered}
$$

We can expand the plane waves into spherical harmonics

$$
\mathrm{e}^{i \mathbf{k} \cdot \mathbf{r}}=\sum_{\ell=0}^{\infty}(2 \ell+1) i^{\ell} P_{\ell}(\cos \theta) j_{\ell}(k r),
$$

where $P_{\ell}$ are Legendre polynomials and $j_{\ell}$ are spherical Bessel functions. Then we will have

$$
\begin{aligned}
& \Psi_{\text {singlet }}(\mathbf{r}, \mathbf{R})=\sqrt{2} \mathrm{e}^{i \mathbf{K} \cdot \mathbf{R}} \sum_{\substack{\ell=0 \\
\text { even } \ell}}^{\infty}(2 \ell+1) i^{\ell} P_{\ell}(\cos \theta) j_{\ell}(k r) \\
& \Psi_{\text {triplet }}(\mathbf{r}, \mathbf{R})=\sqrt{2} \mathrm{e}^{i \mathbf{K} \cdot \mathbf{R}} \sum_{\substack{\ell=1 \\
\text { odd } \ell}}^{\infty}(2 \ell+1) i^{\ell} P_{\ell}(\cos \theta) j_{\ell}(k r) .
\end{aligned}
$$

We can define spin-resolved pair-distribution functions for the uniform electron gas, $g_{\uparrow \uparrow}(r)$ and $g_{\uparrow \downarrow}(r)$, corresponding to parallel- and antiparallel-spin interactions, and such that for the unpolarized gas

$$
g=\frac{1}{2}\left(g_{\uparrow \uparrow}+g_{\uparrow \downarrow}\right) .
$$

They can be related to the formulas just derived by simple considerations: (i) if we select at random a pair of electrons in the uniform gas, there is a probability $p(k)$ that they have relative momentum $k=\left|\mathbf{k}_{2}-\mathbf{k}_{1}\right| / 2$, (ii) the probability for the singlet state is $\frac{1}{4}$ and the probability for the triplet state is $\frac{3}{4}$, (iii) $\frac{1}{3}$ of the triplet state contributes to the antiparallel-spin correlations and $\frac{2}{3}$ of it to the parallel-spin correlations. So, we simply have

$$
\begin{aligned}
& g_{\uparrow \downarrow}(r)=\frac{1}{2}\left\langle\left|\Psi_{\text {singlet }}(\mathbf{r})\right|^{2}\right\rangle+\frac{1}{2}\left\langle\left|\Psi_{\text {triplet }}(\mathbf{r})\right|^{2}\right\rangle \\
& g_{\uparrow \uparrow}(r)=\left\langle\left|\Psi_{\text {triplet }}(\mathbf{r})\right|^{2}\right\rangle,
\end{aligned}
$$

where \langle\rangle denotes average over $p(k)$ and over the solid angle. Performing the spherical average over the solid angle, we obtain:

$$
\begin{aligned}
& g_{\uparrow \downarrow}(r)=\sum_{\ell=0}^{\infty}(2 \ell+1)\left\langle j_{\ell}^{2}(k r)\right\rangle \\
& g_{\uparrow \uparrow}(r)=2 \sum_{\substack{\ell=1 \\
\text { odd } \ell}}^{\infty}(2 \ell+1)\left\langle j_{\ell}^{2}(k r)\right\rangle .
\end{aligned}
$$

Eq. (6) immediately gives the exact result for a noninteracting gas, i.e., $g_{\uparrow \downarrow}(r)=1$ for each $r$. To obtain the noninteracting $g_{\uparrow \uparrow}(r)$ from Eq. (7), we need to average over $k$. In the noninteracting electron gas, the probability distribution $p(k)$ for $k=\frac{1}{2}\left|\mathbf{k}_{2}-\mathbf{k}_{1}\right|$ can be obtained geometrically by considering two three-dimensional vectors $\mathbf{k}_{1}$ and $\mathbf{k}_{2}$ with $0 \leq\left|\mathbf{k}_{1(2)}\right| \leq k_{F}$, where $k_{F}$ is the Fermi wavevector. The probability $p(k)$ is thene6.27

$$
p(k)=24 \frac{k^{2}}{k_{F}^{3}}-36 \frac{k^{3}}{k_{F}^{4}}+12 \frac{k^{5}}{k_{F}^{6}},
$$

with $k$ ranging from 0 to $k_{F}$. $(p(k)$ vanishes at the endpoints of its domain, maximizes around the middle, and integrates to 1.) Then we find analytically that Eq. (7) gives the correct result for the non-interacting uniform gas. Numerically, in the range $0 \leq r / r_{s} \leq 3$ and with a truncation of the infinite sum over $\ell$ at $\ell_{\max }=7$, Eq. (7) reproduces the known exchange-only $g_{\uparrow \uparrow}$ within an accuracy of $10^{-6}$.

With these simple concepts in mind, we can now proceed to compute an interacting pair-distribution function by introducing a suitable electron-electron potential which describes the interactions in a uniform electron gas. Then we just have to replace the spherical Bessel functions $j_{\ell}$ in Eqs. (6) and (7) with the functions $R_{\ell}$, solutions of the radial Schrödinger equation with the chosen two-body potential. Unless this potential is very sophisticated, such a treatment will fail to describe long-range correlations, which are mainly governed by collective modes, and will fail to satisy the particleconservation sum rule on $g_{\sigma \sigma^{\prime}}(r)$ (Eq. (47) of Ref.3).

\section{SOLUTION USING THE OVERHAUSER SCREENED COULOMB POTENTIAL}

Overhauser 18 proposed a simple and reasonable model for the screened Coulomb repulsion $V(r)$ in the uniform electron gas. In the standard uniform-electron-gas model, a rigid positively-charged background maintains electrical neutrality. Thus Overhauser took the sphere of volume $n^{-1}$ as the boundary within which the screening charge density is ne and outside of which it is zero. This is equivalent to assuming that the probability of finding three electrons in a sphere of radius $r_{s}$ is exactly zero, an assumption which is nearly true. In fact, numerical estimates of this probability for an interacting electron gas show that it is indeed small.27 (At $r_{s}=5$ the ratio between the probabilities of finding three and two electrons in the same sphere of radius $r_{s}$ is about $1 / 11$; for larger $r_{s}$ this ratio is lower, and for smaller $r_{s}$ it is higher, being about $1 / 7$ at $r_{s}=0$.)

Thus, for interelectronic distances $r<r_{s}$ we expect the Overhauser potential to be close to the true potential felt by an electron moving in a uniform electron gas when another electron is fixed at the origin. In the region $r>$ $r_{s}$ the potential is set to zero, and so is not expected to be reliable. 


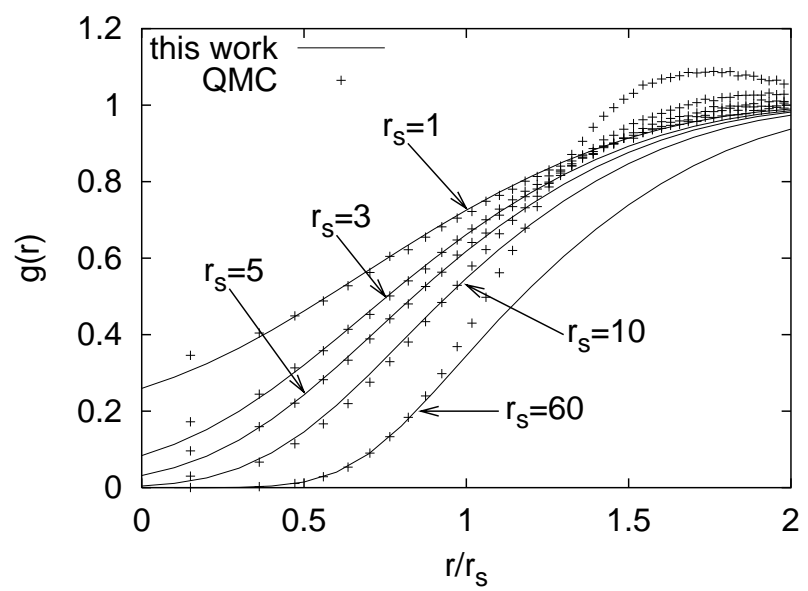

FIG. 1: Pair-distribution functions $g(r)$ fpr the uniform electron gas computed with the Overhauser 18 potential for different values of the electron-density parameter Also shown are the Quantum Monte Carlo data from Ref.18.

We also expect to have results that become more accurate as the density decreases, since the probability of having three electrons in the same sphere of radius $r_{s}$ becomes lower and lower. Finally, at high and intermediate densities our results will be much closer to the true $g(r)$ for antiparallel-spin correlations than for parallelspin ones. When two electrons of opposite spins are in the same sphere of radius $r_{s}$, a third electron is excluded from the sphere because of both the Pauli principle and the Coulomb repulsion. For a pair of parallel-spin electrons, only the Coulomb repulsion prevents a third electron of opposite spin from entering the sphere of radius $r_{s}$, a mechanism which becomes less efficient as the density (and thus the kinetic energy) increases.

In atomic units, the Overhauser potential is given by

$$
\begin{aligned}
& V(r)=\frac{1}{r_{s}}\left(\frac{r_{s}}{r}+\frac{r^{2}}{2 r_{s}^{2}}-\frac{3}{2}\right) \quad\left(r \leq r_{s}\right) \\
& V(r)=\quad 0 \quad\left(r>r_{s}\right) .
\end{aligned}
$$

Defining $s=r / r_{s}, q=k r_{s}$ and $u_{\ell}(s)=q s R_{\ell}(s)$, the corresponding radial Schrödinger equation reads

$$
\begin{aligned}
& {\left[\frac{d^{2}}{d s^{2}}-\frac{\ell(\ell+1)}{s^{2}}+q^{2}-r_{s}\left(\frac{1}{s}+\frac{s^{2}}{2}-\frac{3}{2}\right)\right] u_{\ell}=0} \\
& {\left[\frac{d^{2}}{d s^{2}}-\frac{\ell(\ell+1)}{s^{2}}+q^{2}\right] u_{\ell}=0 \quad(s>1) .}
\end{aligned}
$$

In order to find $u_{\ell}(s)$, we proceed as follows. For $0 \leq s<$ 1 , we expand $u_{\ell}$ in a power series:

$$
u_{\ell}(s)=\alpha_{\ell} \sum_{n=\ell+1}^{\infty} c_{n}^{(\ell)} s^{n} \quad(0 \leq s<1) .
$$

Inserting this expansion into the corresponding radial equation, we find a recursion relation between the co- efficients $c_{n}^{(\ell)}$,

$$
\begin{array}{ll}
c_{n}^{(\ell)}=\frac{\frac{r_{s}}{2} c_{n-4}^{(\ell)}-\left(\frac{3}{2} r_{s}+q^{2}\right) c_{n-2}^{(\ell)}+r_{s} c_{n-1}^{(\ell)}}{(n+\ell)(n-\ell-1)} & (n>\ell+1) \\
c_{\ell+1}^{(\ell)}=1, & c_{n}^{(\ell)}=0 \quad(n \leq \ell) .
\end{array}
$$

For $s>1$ the solution is qs times the spherical Bessel function $j_{\ell}$ with a phase shift $z_{\ell}$. It can be usefully written as

$$
\begin{aligned}
u_{\ell}(s)= & q s\left[f_{\ell}(q s) \sin \left(q s-z_{\ell}\right)+(-1)^{\ell+1} f_{-\ell-1}(q s) \times\right. \\
& \left.\cos \left(q s-z_{\ell}\right)\right] \quad(s>1),
\end{aligned}
$$

where $f_{j}(x)$ are given by

$$
\begin{array}{r}
f_{0}(x)=x^{-1} \quad f_{1}(x)=x^{-2} \\
f_{j-1}(x)+f_{j+1}(x)=(2 j+1) x^{-1} f_{j}(x) .
\end{array}
$$

Equation (13) is properly normalized, since the noninteracting case of Eqs. (6) and (7) is exactly recovered when $z_{\ell}=0$. By matching $u_{\ell}(s)$ and its first derivative at $s=1$, we can find $\alpha_{\ell}$ and the phase shift $z_{\ell}$. They are given by

$$
\begin{aligned}
& \frac{\alpha_{\ell}^{2}}{q^{2}}=\frac{q^{2}\left[f_{\ell}(q) f_{-\ell}(q)+f_{\ell-1}(q) f_{-\ell-1}(q)\right]^{2}}{\mathcal{S}_{\ell}\left(q, r_{s}\right)^{2}+\mathcal{C}_{\ell}\left(q, r_{s}\right)^{2}} \\
& \tan \left(q-z_{\ell}\right)=(-1)^{\ell} \frac{\mathcal{S}_{\ell}\left(q, r_{s}\right)}{\mathcal{C}_{\ell}\left(q, r_{s}\right)}
\end{aligned}
$$

where

$$
\begin{array}{r}
\mathcal{S}_{\ell}=q f_{-\ell}(q) B_{\ell}+f_{-\ell-1}(q) A_{\ell} \\
\mathcal{C}_{\ell}=f_{\ell}(q) A_{\ell}-q f_{\ell-1}(q) B_{\ell} \\
A_{\ell}=A_{\ell}\left(q, r_{s}\right)=\sum_{n=\ell+1}^{\infty} c_{n}^{(\ell)}(n+\ell) \\
B_{\ell}=B_{\ell}\left(q, r_{s}\right)=\sum_{n=\ell+1}^{\infty} c_{n}^{(\ell)} .
\end{array}
$$

With $8\left(\ell_{\max }=7\right)$ partial waves and the infinite sum over $n$ in Eqs. (11), (19) and (20) truncated at $n_{\max } \sim 20$, we reach accurate convergence for $0 \leq r / r_{s} \leq 2$.

We thus obtain smooth radial functions $R_{\ell}\left(s, r_{s}, q\right)$ to replace the functions $j_{\ell}$ in Eqs. (6) and (7). Then we have to average over $q$ to obtain $g_{\sigma \sigma^{\prime}}\left(s, r_{s}\right)$. We used the probability distribution $p(q)$ corresponding to an unpolarized noninteracting uniform electron gas, i.e., we took Eq. (8) with $k_{F}=\frac{1}{r_{s}}\left(\frac{9 \pi}{4}\right)^{1 / 3}$,

$$
p(q)=\frac{16}{3 \pi} q^{2}\left[2-\left(\frac{12}{\pi}\right)^{1 / 3} q+\frac{4}{9 \pi} q^{3}\right],
$$

with $q$ ranging from 0 to $(9 \pi / 4)^{1 / 3}$. The $q$-dependence of our results is rather weak, so we expect to have no significant change if we use an interacting momentum distribution instead of the one of Eq. (21).

The results for the total $g(r)$ are shown in Fis 11, together with the newest QMC results from Ref.16. We 
find $g(r)$ in accurate agreement with the QMC data for $0.5 \lesssim r / r_{s} \lesssim 1$ for a wide range of electron densities. In the shortest-range region, $r / r_{s} \lesssim 0.5$, the QMC data are known to suffer large errors and are not so reliable. (In this region there is in fact a significant discrepancy between the data from Ref. 6 and those from Ref 13 .) We believe that for $r / r_{s} \lesssim 0.5$ the present treatment provides results much closer to the true $g(r)$. As said, for $r / r_{s}>1$ the results obtained with the Overhauser potential are not reliable.

\section{SHORT-RANGE COEFFICIENTS OF THE PAIR-DISTRIBUTION FUNCTION}

The small-s expansion of the spin-resolved $g_{\uparrow \downarrow}$ and $g_{\uparrow \uparrow}$ (where $s=r / r_{s}$ ),

$$
\begin{aligned}
& g_{\uparrow \downarrow}\left(s, r_{s}\right)=a_{0}^{\uparrow \downarrow}\left(r_{s}\right)+a_{1}^{\uparrow \downarrow}\left(r_{s}\right) s+a_{2}^{\uparrow \downarrow}\left(r_{s}\right) s^{2}+O(s(2) 2) \\
& g_{\uparrow \uparrow}\left(s, r_{s}\right)=a_{2}^{\uparrow \uparrow}\left(r_{s}\right) s^{2}+a_{3}^{\uparrow \uparrow}\left(r_{s}\right) s^{3}+O\left(s^{4}\right),
\end{aligned}
$$

obtained by solving the Overhauser equation, has coefficients

$$
\begin{aligned}
& a_{0}^{\uparrow \downarrow}=\left\langle\frac{\alpha_{0}^{2}}{q^{2}}\right\rangle \\
& a_{1}^{\uparrow \downarrow}=r_{s}\left\langle\frac{\alpha_{0}^{2}}{q^{2}}\right\rangle=r_{s} a_{0}^{\uparrow \downarrow} \\
& a_{2}^{\uparrow \downarrow}=\frac{r_{s}}{12}\left(5 r_{s}-6\right)\left\langle\frac{\alpha_{0}^{2}}{q^{2}}\right\rangle-\frac{\left\langle\alpha_{0}^{2}\right\rangle}{3}+3\left\langle\frac{\alpha_{1}^{2}}{q^{2}}\right\rangle \\
& a_{2}^{\uparrow \uparrow}=6\left\langle\frac{\alpha_{1}^{2}}{q^{2}}\right\rangle \\
& a_{3}^{\uparrow \uparrow}=3 r_{s}\left\langle\frac{\alpha_{1}^{2}}{q^{2}}\right\rangle=\frac{r_{s}}{2} a_{2}^{\uparrow \uparrow},
\end{aligned}
$$

where the $\alpha_{\ell}$ are given by Eq. (15) and \langle\rangle means average over $p(q)$ of Eq. (21). Fquations (25) and (28) are the known cusp conditions, 128 due to the dominance for $r \rightarrow$ 0 of the term $1 / r$ in the Schrödinger equation.

Equation (24) gives the value of the pair-distribution function at zero-interelectronic distance, $g(0)=a_{0}^{\uparrow \downarrow} / 2$. In Fig. 2 we compare the present result with other estimates $g^{f} g(0)$ : (i) two approximate solutions that Overhauser 18 obtained from his model potential after setting $q=0$ in the radial Schrödinger equation for $s \leq 1$; (ii) the Yasuharat7 electron-electron ladder evaluation; (iii) the Perdew-Wange (PW92) formula, and (iv) the result extrapolated from QMC data 16 by Gori-Giorgi, Sacchetti and Bachelet (GSB). The value of $g(0)$ has been multiplied by the density parameter $r_{s}$ in order to amplify the differences between the curves, which are mainly located at low densities. We see that the first Overhauser formula, obtained from a first iteration, is quite far from the "exact" solution of his model presented in this work. His second formula, derived by a second iteration, is much closer to our solution (within $5 \%$ at $r_{s}=2$, $50 \%$ at $\left.r_{s}=10\right)$. The Yasuhara electron-electron ladder

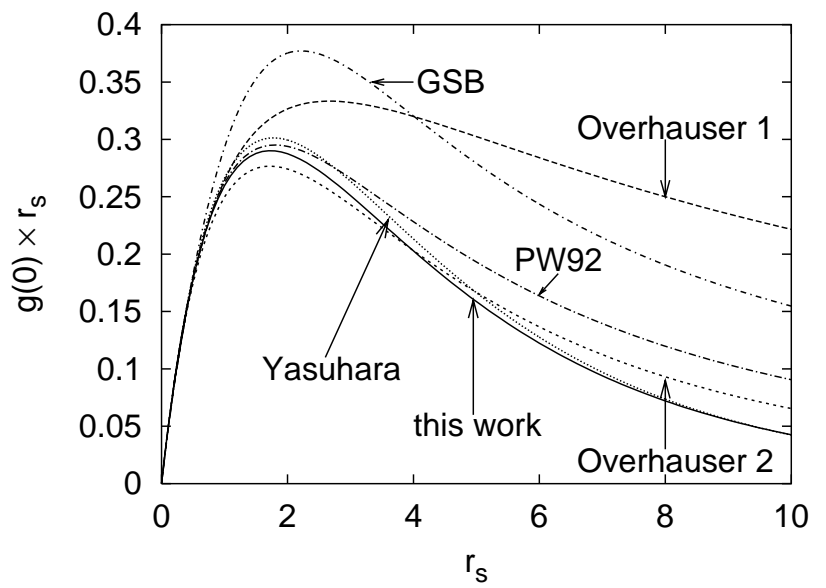

FIG. 2: Pair-distribution function at zero-interelectronic distance multiplied by the density parameter $r_{s}$. The present result (exact solution of the Overhauser model) is compared with the two Pverhauser formulas 18 ( 1 and 2 iterations), with the Yasuhara17 eleftron-electron ladder evaluation, with the Perdew and Wang (PH92) formula, and with the extrapolation from $\mathrm{MC}$ data 16 obtained by Gori-Giorgi, Sacchetti and Bachelet5 (GSB).

evaluation is the closest to our result for $r_{s} \gtrsim 5$ (e.g., the difference is only $0.6 \%$ at $r_{s}=10$ ). This is not surprising since both calculations are more reliable at lower densities. The PW92 formula is mainly a Padé approximation of the Yasuhara values; it is not very accurate at densities $r_{s} \gtrsim 4$, where the electron-electron ladder $g(0)$ goes to zero as $19 r_{s}^{3 / 2} \mathrm{e}^{-A \sqrt{r_{s}}}$, while the PW92 Padé approximant goes as $r_{s}^{-3}$. However, the PW92 formula reproduces quite accurately the Yasuhara values at metallic densities, and has the advantage of being very simple and handy for many purposes. The extrapolated GSB value is, for $r_{s} \gtrsim 0.7$, much higher than the curve obtained in the present work, as expected from the results of Fig. 1, in which our $g(r)$ curves always lie below the QMC data for $r / r_{s} \lesssim 0.3$.

The high-density limit of our $a_{0}^{\uparrow \downarrow}\left(r_{s}\right)$ is

$$
a_{0}^{\uparrow \downarrow}\left(r_{s} \rightarrow 0\right)=1-0.684 r_{s}+O\left(r_{s}^{2}\right),
$$

which differs from the exact one, $20291-0.7317 r_{s}$, by only $6.5 \%$ in the coefficient of $r_{s}$ and is slightly better than the high-density limit of the Yasuharal $17 a_{0}^{\uparrow \downarrow}$, $1-0.663 r_{s} .19$ We can conclude that the value of $g(0)$ obtained by solving the Overhauser model is very reliable: it almost recovers the exact high-density limit, and agrees in the low-density limit with the electron-electron ladder evaluation of Yasuhara. In the latter limit, where the electrons become strictly correlated 2- $a_{2}^{\uparrow \downarrow}$ and $a_{2}^{\uparrow \uparrow}$ tend to zero.

Our results for $g(0)$ can be usefully parametrized with the following form

$$
a_{0}^{\uparrow \downarrow}\left(r_{s}\right)=\left(1-B r_{s}+C r_{s}^{2}+D r_{s}^{3}+E r_{s}^{4}\right) \mathrm{e}^{-d r_{s}} .
$$



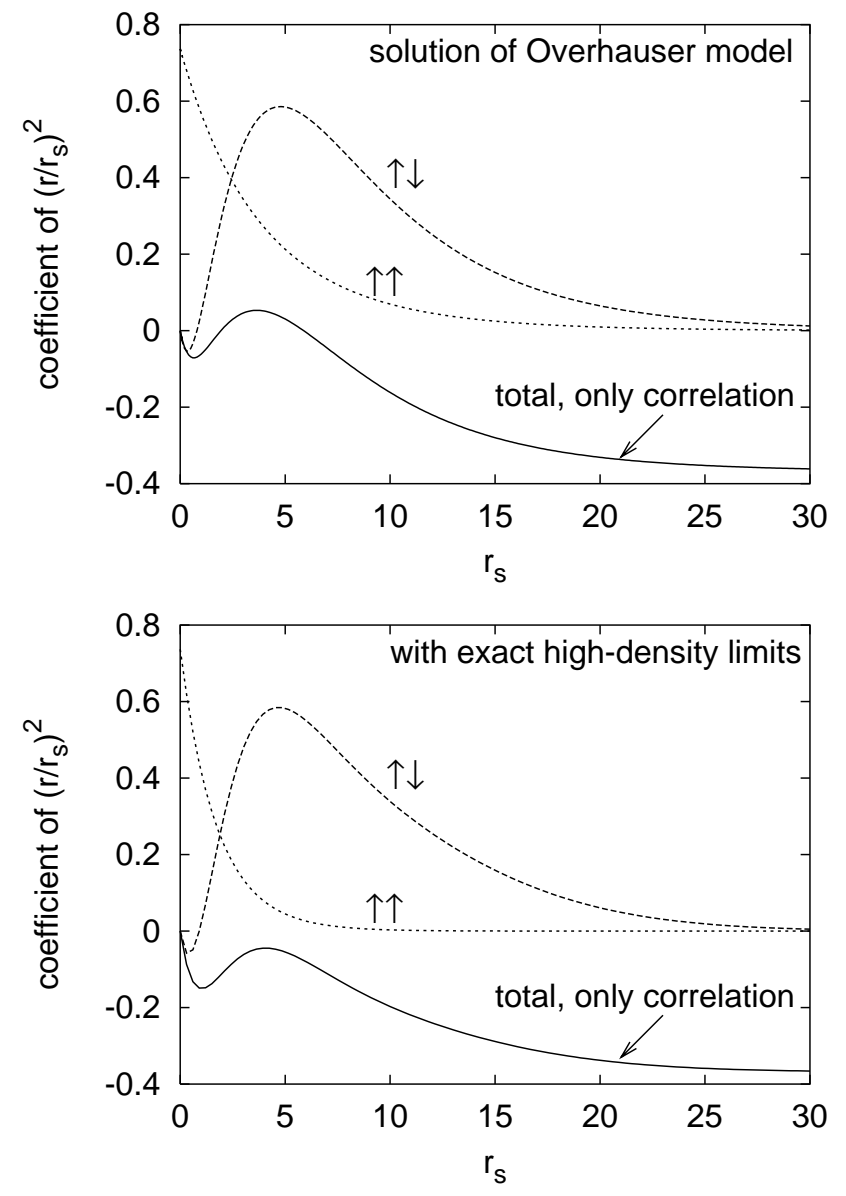

FIG. 3: Coefficients of $\left(r / r_{s}\right)^{2}$ in the small- $r$ expansion of the pair-distribution function. The parallel- and antiparallel-spin contributions are separately shown. The total coefficient for the correlation part, i.e., $a_{2}^{c}=\frac{1}{2}\left(a_{2}^{\uparrow \downarrow}+a_{2}^{\uparrow \uparrow}\right)-\frac{1}{10}\left(\frac{9 \pi}{4}\right)^{2 / 3}$, is also reported. Upper panel: solution of the Overhauser model; lower panel: interpolation formulas including the exact highdensity limits.

We constrain our fit to reproduce the exact high-density limit, in order to be as close as possible to the true $g(0)$. Thus we fix $B=0.7317-d$. A best fit of the remaining 4 free parameters to our result for $r_{s} \leq 10$ gives $C=$ $0.08193, D=-0.01277, E=0.001859, d=0.7524$. Then $B=-0.0207$. On the scale of Fig. 2, the fit error is invisible.

As far as we know, the coefficients $a_{2}^{\uparrow \downarrow}$ and $a_{2}^{\uparrow \uparrow}$, given by Eqs. (26) and (27), are presented in this work for the first time. The good performance of $g(0)$ obtained with the Overhauser potential, together with the agreement of $g(r)$ with the QMC data for $0.5 \lesssim r / r_{s} \lesssim 1$, suggest that our calculation of these coefficients is quantitatively reliable. In the upper panel of Fig. B, we report $a_{2}^{\uparrow \downarrow}$ and $a_{2}^{\uparrow \uparrow}$ as a function of $r_{s}$. While the parallel-spin part shows a predictable dependence on $r_{s}$, decreasing monotonically from the noninteracting value $\frac{1}{5}\left(\frac{9 \pi}{4}\right)^{2 / 3}$ to zero as $r_{s}$ increases, the $\uparrow \downarrow$ part presents at first glance a non-intuitive dependence on the density. However, if we consider the known high- and low-density behavior of $a_{2}^{\uparrow \downarrow}$, we see that we should expect a function which is qualitatively similar to the one we obtained. In fact, in the $r_{s} \rightarrow 0$ limit $a_{2}^{\uparrow \downarrow}$ is known to approach zero from negative values,, while in the $r_{s} \rightarrow \infty$ limit we expect $a_{2}^{\uparrow \downarrow}$ to approach zero asymptotically from positive values, in order to fulfill the positivity constraint on $g_{\uparrow \downarrow}(r)$. Thus, $a_{2}^{\uparrow \downarrow}$ must have a minimum and a maximum, and must cross zero in between, as does our result.

The high-density limit of our $s^{2}$ coefficients is

$$
\begin{aligned}
& a_{2}^{\uparrow \downarrow}\left(r_{s} \rightarrow 0\right)=-0.34 r_{s}+O\left(r_{s}^{2}\right) \\
& a_{2}^{\uparrow \uparrow}\left(r_{s} \rightarrow 0\right)=\frac{1}{5}\left(\frac{9 \pi}{4}\right)^{2 / 3}-0.192 r_{s}+O\left(r_{s}^{2}\right) .
\end{aligned}
$$

The $\uparrow \uparrow \downarrow$ value is in excellent agreement with the exact one, $-0.3356 r_{s}$, while the $\uparrow \uparrow$ coefficient of $r_{s}$ in the r.h.s. of Eq. (32) is $54 \%$ smaller than the exact one, -0.422 ; see also Ref.t. As said, in the high-density limit the assumption of zero probability of having a third electron in the same sphere of radius $r_{s}$ can be nearly true only for antiparallel spin electrons, since in this case the Pauli principle keeps the third electron away. So, our $a_{2}^{\uparrow \downarrow}$ should be very close to the true one, while the $\uparrow \uparrow$ shortrange correlations are underestimated at higher densities in the present treatment (i.e., the true $a_{2}^{\uparrow \uparrow}$ deviates more from the noninteracting value $\frac{1}{5}\left(\frac{9 \pi}{4}\right)^{2 / 3}$, so it is lower than our result). However, $a_{2}^{\uparrow \uparrow}$ has a very simple dependence on $r_{s}$, so, as we shall see, it is very easy to write down an interpolation formula between the high- and the low-density limits.

In the upper panel of Fig. 3 we also show $a_{2}^{c}$, the total $\left(r / r_{s}\right)^{2}$ coefficient of the correlation part of $g(r)$, i.e., $\frac{1}{2}\left(a_{2}^{\uparrow \downarrow}+a_{2}^{\uparrow \uparrow}\right)$ minus the noninteracting value $\frac{1}{10}\left(\frac{9 \pi}{4}\right)^{2 / 3}$. We find that $a_{2}^{c}$ is positive in the density range $1.94<$ $r_{s}<5.88$, and negative elsewhere. However, we believe that the positive region is a consequence of underestimating the $\uparrow \uparrow$ correlations.

Our results are very well reproduced by the simple formulas

$$
\begin{aligned}
& a_{2}^{\uparrow \downarrow}=\left(-\beta_{a} r_{s}+\gamma_{a} r_{s}^{2}+\delta_{a} r_{s}^{3}+\epsilon_{a} r_{s}^{4}\right) \mathrm{e}^{-d_{a} r_{s}} \\
& a_{2}^{\uparrow \uparrow}=\frac{1}{5}\left(\frac{9 \pi}{4}\right)^{2 / 3}\left(1-\beta_{p} r_{s}+\gamma_{p} r_{s}^{2}\right) \mathrm{e}^{-d_{p} r_{s}}
\end{aligned}
$$

where $\beta_{a}=0.32, \gamma_{a}=0.4069, \delta_{a}=-0.04455, \epsilon_{a}=$ $0.003064, d_{a}=0.4235, \beta_{p}=0.01624, \gamma_{p}=0.00264, d_{p}=$ 0.2456 .

However, as said, the $\uparrow \uparrow$ correlations are underestimated. We can use the formulas of Ess. (33) and (34) to recover the exact high-density limits by simply changing the parameters $\beta_{a}$ and $d_{p}$. The new values are $\beta_{a}=0.3356, d_{p}=0.5566$. In this way, the $\uparrow \downarrow$ coefficient is basically unchanged, while the $\uparrow \uparrow$ is lowered because of the exact slope at $r_{s}=0$, and should be much closer to the true one. The new $a_{2}$ coefficients are shown in the lower panel of Fig. 3, together with the total coefficient for the correlation part. We see that now $a_{2}^{c}$ is always negative. 
Ziesche 30 has proposed $1-5\left(\frac{9 \pi}{4}\right)^{-2 / 3} a_{2}^{\uparrow \uparrow}$ as a measure of correlation strength, varying monotonically from 0 for $r_{s} \rightarrow 0$ to 1 for $r_{s} \rightarrow \infty$.

\section{COUPLING-CONSTANT AVERAGE}

The coupling-constant average 31 of the pairdistribution function for the uniform electron gas is given by

$$
\bar{g}\left(s, r_{s}\right)=\frac{1}{r_{s}} \int_{0}^{r_{s}} g\left(s, r_{s}^{\prime}\right) d r_{s}^{\prime},
$$

where $s$ is kept fixed. The function $\bar{g}$ is important because it can account for the kinetic energy of correlation. Its short-range coefficients can be computed by integrating the simple formulas of Eqs. (30), (33), and (34):

$$
\begin{aligned}
\bar{a}_{0}^{\uparrow \downarrow}= & \frac{1}{r_{s}}\left[\left(-\bar{A}+\bar{B} r_{s}+\bar{C} r_{s}^{2}+\bar{D} r_{s}^{3}+\bar{E} r_{s}^{4}\right) \mathrm{e}^{-d r_{s}}\right. \\
& +\bar{A}] \\
\bar{a}_{1}^{\uparrow \downarrow}= & \frac{1}{r_{s}}\left[\left(-\bar{A}_{1}+\bar{B}_{1} r_{s}+\bar{C}_{1} r_{s}^{2}+\bar{D}_{1} r_{s}^{3}+\bar{E}_{1} r_{s}^{4}\right.\right. \\
& \left.\left.+\bar{F}_{1} r_{s}^{5}\right) \mathrm{e}^{-d r_{s}}+\bar{A}_{1}\right] \\
\bar{a}_{2}^{\uparrow \downarrow}= & \frac{1}{r_{s}}\left[\left(-A_{a}+B_{a} r_{s}+C_{a} r_{s}^{2}+D_{a} r_{s}^{3}+E_{a} r_{s}^{4}\right) \mathrm{e}^{-d_{a} r_{s}}\right. \\
& \left.+A_{a}\right] \\
\bar{a}_{2}^{\uparrow \uparrow}= & \frac{1}{5}\left(\frac{9 \pi}{4}\right)^{2 / 3} \frac{1}{r_{s}}\left[\left(-A_{p}+B_{p} r_{s}+C_{p} r_{s}^{2}\right) \mathrm{e}^{-d_{p} r_{s}}\right. \\
& \left.+A_{p}\right]
\end{aligned}
$$

where for $\bar{a}_{0}^{\uparrow \downarrow}$ we obtain $\bar{A}=1.696, \bar{B}=-0.2763, \bar{C}=$ $-0.09359, \bar{D}=0.003837, \bar{E}=-0.002471$, and for $\bar{a}_{1}^{\uparrow \downarrow}$ we obtain $\bar{A}_{1}=3.356, \bar{B}_{1}=-2.525, \bar{C}_{1}=-0.45, \bar{D}_{1}=$ $-0.106, \bar{E}_{1}=0.000553, \bar{F}_{1}=-0.00247$. Equation (37) has been obtained by integrating $r_{s} a_{0}^{\uparrow \downarrow}\left(r_{s}\right)$, according to the cusp condition. In this way, we are able to give shortrange coefficients which exactly satisfy the modified cusp condition for $\bar{g}(r)$. (In the Perdew-Wang $\overline{3}$ model for $\bar{g}$, the cusp condition is accurately but not exactly satisfied.)

For the $s^{2}$ coefficients, using the values corresponding to the exact high-density limit formulas, we have: $A_{a}=$ 5.9313, $B_{a}=-2.5119, C_{a}=-0.6997, D_{a}=0.03686$, $E_{a}=-0.007235, d_{a}=0.4235, A_{p}=1.775, B_{p}=0.0121$, $C_{p}=-0.00474, d_{p}=0.5566$.

\section{SPIN-POLARIZED GAS}

The spin-polarized electron gas is characterized by the parameter $\zeta=\left(n_{\uparrow}-n_{\downarrow}\right) / n$, where $n_{\uparrow}$ and $n_{\downarrow}$ are the densities of spin-up and -down electrons. The pairdistribution function averaged over $\uparrow \uparrow, \downarrow \downarrow$, and $\uparrow \downarrow$ pairs is then

$$
\begin{aligned}
g\left(r, r_{s}, \zeta\right)= & \left(\frac{1+\zeta}{2}\right)^{2} g_{\uparrow \uparrow}\left(r, r_{s}, \zeta\right)+\left(\frac{1-\zeta}{2}\right)^{2} g_{\downarrow \downarrow}\left(r, r_{s}, \zeta\right) \\
& +\frac{\left(1-\zeta^{2}\right)}{2} g_{\uparrow \downarrow}\left(r, r_{s}, \zeta\right) .
\end{aligned}
$$

Here we shall motivate and use an approximate spin scaling relation for the short-range part of $g$ :

$$
g_{\sigma \sigma^{\prime}}\left(r, r_{s}, \zeta\right)=g_{\sigma \sigma^{\prime}}\left(r, r_{s}^{\sigma \sigma^{\prime}}(\zeta), \zeta=0\right),
$$

where $r_{s}^{\uparrow \uparrow}=r_{s}(1+\zeta)^{-1 / 3}, r_{s}^{\downarrow \downarrow}=r_{s}(1-\zeta)^{-1 / 3}$, and $r_{s}^{\uparrow \downarrow}=2 r_{s} /\left[(1+\zeta)^{1 / 3}+(1-\zeta)^{1 / 3}\right]$.

In the present treatment, information about the spin polarization of the system enters when we average over the probability $p(q)$. For a spin-unpolarized $(\zeta=0)$ gas, the correct $p(q)$ is the one of Eq. (21). For a fully polarized $(\zeta=1)$ gas, we can similarly obtain $p(q)$ from Eq. (8) by using the proper relation between $k_{F}$ and $r_{s}$, i.e., $k_{F}^{\zeta=1}=\frac{1}{r_{s}}\left(\frac{9 \pi}{2}\right)^{1 / 3}=2^{1 / 3} k_{F}^{\zeta=0}$. For a partiallypolarized gas $(0<\zeta<1)$, we have to distinguish between the parallel- and the antiparallel-spin cases. In the parallel-spin case, we can still obtain $p(q)$ from Eq. (8) by using $k_{F \uparrow}=(1+\zeta)^{1 / 3} k_{F}^{\zeta=0}$ for $\uparrow \uparrow$ interactions, and $k_{F \downarrow}=(1-\zeta)^{1 / 3} k_{F}^{\zeta=0}$ for $\downarrow \downarrow$ interactions. This means that we can obtain $p_{\zeta}^{\uparrow \uparrow}(q)$ and $p_{\zeta}^{\downarrow \downarrow}(q)$ by rescaling $p(q)$ of Eq. (21) as follows:

$$
\begin{gathered}
p_{\zeta}^{\uparrow \uparrow}(q)=(1+\zeta)^{-1 / 3} p\left[q(1+\zeta)^{-1 / 3}\right] \\
p_{\zeta}^{\downarrow \downarrow}(q)=(1-\zeta)^{-1 / 3} p\left[q(1-\zeta)^{-1 / 3}\right],
\end{gathered}
$$

with $q$ ranging from 0 to $\left[\frac{9 \pi}{4}(1+\zeta)\right]^{1 / 3}$ for the $\uparrow \uparrow$ case and from 0 to $\left[\frac{9 \pi}{4}(1-\zeta)\right]^{1 / 3}$ for the $\downarrow \downarrow$ case. In the case of a pair of antiparallel-spin electrons, we have to compute geometrically the probability distribution for a variable $k=\frac{1}{2}\left|\mathbf{k}_{2}-\mathbf{k}_{1}\right|$ when $0 \leq\left|\mathbf{k}_{1}\right| \leq k_{F \downarrow}$ and $0 \leq\left|\mathbf{k}_{2}\right| \leq k_{F \uparrow}$. This gives

$$
\begin{aligned}
p_{\zeta}^{\uparrow \downarrow}(k) & =24 \frac{k^{2}}{k_{F \uparrow}^{3}} \quad\left(\text { for } 0 \leq k \leq k_{-}\right) \\
& =-\frac{9}{4} \frac{\left(k_{F \uparrow}^{2}-k_{F \downarrow}^{2}\right)^{2}}{k_{F \uparrow}^{3} k_{F \downarrow}^{3}} k+12 \frac{\left(k_{F \uparrow}^{3}+k_{F \downarrow}^{3}\right)}{k_{F \uparrow}^{3} k_{F \downarrow}^{3}} k^{2}-18 \frac{\left(k_{F \uparrow}^{2}+k_{F \downarrow}^{2}\right)}{k_{F \uparrow}^{3} k_{F \downarrow}^{3}} k^{3} \\
& +\frac{12}{k_{F \uparrow}^{3} k_{F \downarrow}^{3}} k^{5} \quad\left(\text { for } k_{-} \leq k \leq k_{+}\right),
\end{aligned}
$$

where $k_{ \pm}=\frac{1}{2}\left(k_{F \uparrow} \pm k_{F \downarrow}\right)$. Our goal is to write down a scaling relation which allows us to use the results obtained for the $\zeta=0$ gas. To do this, first we have to write down an approximate $p_{\zeta}^{\uparrow \downarrow}(q)$ obtained by scaling $p(q)$. A reasonable choice, having correct limits and normalization, is

$$
p_{\zeta}^{\uparrow \downarrow}(q) \approx \frac{2}{(1+\zeta)^{1 / 3}+(1-\zeta)^{1 / 3}} p\left[\frac{2 q}{(1+\zeta)^{1 / 3}+(1-\zeta)^{1 / 3}}\right],
$$

with $q$ varying from 0 to $\left(\frac{9 \pi}{4}\right)^{1 / 3} \frac{\left[(1+\zeta)^{1 / 3}+(1-\zeta)^{1 / 3}\right]}{2}$.

In order to use the results obtained for the unpolarized gas, we now consider that $q=k r_{s}$. Scaling $q$ according to Eqs. (42), (43) and (45) can thus be approximately equivalent to rescaling $r_{s}$. This is exact if the Overhauser radial Schrödinger equation is changed in the same way when we replace $q$ with $\mu q$ and when we replace $r_{s}$ with $r_{s} / \mu$. In the latter case, one also has to consider that the variable $s=r / r_{s}$ is changed into $s^{\prime}=\mu r / r_{s}=\mu s$. 
One can easily check that this scaling property is satisfied by the equation with the potential set to zero $(s>1)$, which means that it is exact at the exchange-only level. For the case of nonzero potential $(0 \leq s \leq 1)$, the scaling condition is satisfied only by the kinetic terms and by the $1 / s$ part of the potential. However, since for small $s$ the $1 / s$ part of the potential is dominant, we expect to obtain rather good results by applying this scaling to the short-range coefficients.

When we apply our scaling relation to the total $s^{2}$ coefficient of $g$, we obtain

$$
\begin{aligned}
& a_{2}\left(r_{s}, \zeta\right)=\left(\frac{1+\zeta}{2}\right)^{2}(1+\zeta)^{2 / 3} a_{2}^{\uparrow \uparrow}\left[r_{s}(1+\zeta)^{-1 / 3}\right]+ \\
&\left(\frac{1-\zeta}{2}\right)^{2}(1-\zeta)^{2 / 3} a_{2}^{\uparrow \uparrow}\left[r_{s}(1-\zeta)^{-1 / 3}\right]+\frac{\left(1-\zeta^{2}\right)}{2} \times \\
& {\left[\frac{(1+\zeta)^{1 / 3}+(1-\zeta)^{1 / 3}}{2}\right]^{2} a_{2}^{\uparrow \downarrow}\left[\frac{2 r_{s}}{(1+\zeta)^{1 / 3}+(1-\zeta)^{1 / 3}}\right],(46) }
\end{aligned}
$$

where the functions $a_{2}^{\uparrow \downarrow}\left(r_{s}\right)$ and $a_{2}^{\uparrow \uparrow}\left(r_{s}\right)$ can be either the ones of Eqs. (33) and (34) or the ones of Eqs. (38) and (39), depending whether one is interested in $g$ or in its coupling-constant average $\bar{g}$. Equation (46) is exact (beyond the Overhauser model) in several limits: for exchange, for parallel-spin correlation in the high-density limit (Eq. (10) of Ref.J), and for the low-density limit. The fact that Eq. (46) is exact for parallel-spin correlations in the $r_{s} \rightarrow 0$ limit suggests that the results for the short-range coefficients of $g(r)$ are not affected by the violation of the scaling relation by the part of the potential which remains finite when $s \rightarrow 0$.

For the $\uparrow \downarrow$ interactions, we have also the error due to the use of an approximate relative-momentum distribution. We can get an idea of this error by applying our spin-scaling relation to $g(0)$, with the result

$g\left(r=0, r_{s}, \zeta\right)=\left(1-\zeta^{2}\right) g\left(r=0, \frac{2 r_{s}}{(1+\zeta)^{1 / 3}+(1-\zeta)^{1 / 3}}, \zeta=0\right)$

Equation (47) is different from that of Marinescu and Quinn32 or Perdew and Wang, 3 obtained by using an exchange-like $\left(r_{s}=0\right) \zeta$-dependence. The high-density limit of $g\left(0, r_{s}, \zeta\right)$ is

$$
g\left(r=0, r_{s} \rightarrow 0, \zeta\right)=\frac{1}{2}\left(1-\zeta^{2}\right)\left[1-\lambda(\zeta) r_{s}\right]
$$

where from Eq. 47)

$$
\lambda(\zeta)=\frac{2 \lambda(\zeta=0)}{(1+\zeta)^{1 / 3}+(1-\zeta)^{1 / 3}} .
$$

In Fig. 14 compare our Eq. (49) with the exact $\lambda(\zeta) / \lambda(\mathrm{Q}), 19$ and with the exchange-like approximation of Refs. 3 and 32 , in which this ratio is 1 . We see that our scaling relation is always closer to the exact $\zeta$-dependence than is the exchange-like one. The maximum error is at $\zeta=1$, where our ratio is 1.587 and the exact one is 1.332 . (By the way, we numerically fonfirm the equivalence of the exact expressions in Refs.

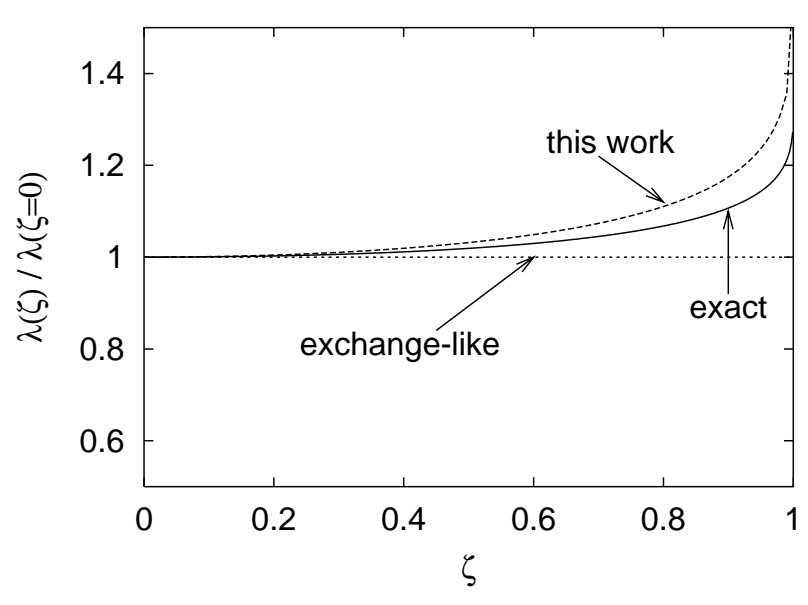

FIG. 4: Spin-polarization dependence of $g(0)$ at high- density. $\lambda(\zeta)$ is defined by Eq. (48). The scaling prqtiction of Eq. (49) is compared to the exact $\zeta$-dependenem, tha and to the exchange-like scaling adopted in Refs 3 and 32 .

\section{CONCLUSIONS AND FUTURE DIRECTIONS}

Our work supports and extends Overhauser's picture of short-range correlations in the uniform electron gas of density $n=3 / 4 \pi r_{s}^{3}$. When two electrons are separated by a distance $r \lesssim r_{s}$, there is very little probability of finding a third electron within a sphere of radius $r_{s}$ about the first (especially in the strong-interaction or large- $r_{s}$ limit). Thus the short-range correlations arise from twoelectron collisions with an effective potential like that of Eq. (9).

We have evaluated the resulting pair distribution $g(r)$, not just for $r=0$ [Fig. 2 and Eq. (30), which confirm the accuracy of the Yasuhara $17 g(0)]$ but for all $r$ (Fig. 1). We find good agreement with Quantum Monte Carlo data in the range $0.5 r_{s} \lesssim r \lesssim r_{s}$, where this data is accurate, while our results are probably more reliable than the Monte Carlo data for $r \lesssim 0.5 r_{s}$.

We have also studied the small- $r$ expansion of the spinresolved pair-distribution functions, Eqs. (22) and (23). The $r_{s}$-dependent coefficients $a_{2}^{\sigma \sigma^{\prime}}\left(r_{s}\right)$ of $\left(r / r_{s}\right)^{2}$ from the Overhauser model have been extracted and then corrected for the known high-density limit 0 [Fig. 3, Eqs. (33) and (34) with corrected $\beta_{a}$ and $\left.d_{p}\right]$. Unlike $g(0)$ and $a_{2}^{\uparrow \uparrow}$, our $a_{2}^{\uparrow \downarrow}$ has a non-monotonic dependence upon $r_{s}$. We have also discussed how to average the short-range coefficients of $g(r)$ over coupling constant [Eqs. (36)-(39)], and how to scale them for relative spin polarization $\zeta \neq 0$ [Eqs. (46) and (47)]. In future work, we plan to use these results to make a new analytic model for the uniform-gas $g(r)$ at all $r$, for use in density functional theory. Unlike currently available models which fail at very small or very large $r_{s}$, this new model will be designed to work over the whole range $0<r_{s}<\infty$, while satisfying more exact constraints. 
Except very close to $r=0$, highly accurate pairdistribution functions for the uniform electron gas can be found from Quantum Monte Carlo simulations, or from the fluctuation-dissipation theorem using 33 RPA like response functions with the Richardson-Ashcroft 34 local-field factor. By supplementing that approach with our small-r expansions of Eqs. 22, and (23), it should be possible to find essentially-exact spin-resolved pairdistribution functions and correlation energies.

Finally, we suspect that this approach can be applied usefully to other systems, including the two-dimensional electron gas 35,36

\section{Acknowledgments}

This work was supported in part by the Fondazione Angelo Della Riccia (Firenze, Italy), and in part by the U.S. National Science Foundation under grant No. DMR98-10620. We are very grateful to A. W. Overhauser for sending us the derivation of Eq. (8) and for many useful comments at an early stage of our work, and to Paul Ziesche for many helpful suggestions.
1 A. K. Rajagopal, J. C. Kimball, and M. Banerjee, Phys. Rev. B 18, 2339 (1978).

2 V. Contini, G. Mazzone, and F. Sacchetti, Phys. Rev. B 33, 712 (1986).

3 J. P. Perdew and Y. Wang, Phys. Rev. B 46, 12947 (1992); 56, 7018 (1997).

4 V. A. Rassolov, J. A. Pople, and M. A. Ratner, Phys. Rev. B 59, 15625 (1999).

${ }^{5}$ P. Gori-Giorgi, F. Sacchetti, and G. B. Bachelet, Phys. Rev. B 61, 7353 (2000).

${ }^{6}$ K. Schmidt, S. Kurth, J. Tao, and J. P. Perdew, Phys. Rev. B 62, 2227 (2000).

7 V. A. Rassolov, J. A. Pople, and M. A. Ratner, Phys. Rev. B 62, 2232 (2000).

8 O. Gunnarsson, M. Jonson, and B. I. Lundqvist, Phys. Lett. A 59, 177 (1976); Phys. Rev. B 20, 3136 (1979).

${ }^{9}$ E. Chacón and P. Tarazona, Phys. Rev. B 37, 4013 (1988).

10 J. P. Perdew, Electronic Structure of Solids '91, edited by P. Ziesche and H. Eschrig (Akademie Verlag, Berlin 1991); J. P. Perdew, K. Burke, and M. Ernzerhof, Phys. Rev. Lett. 77, 3865 (1996); 78, 1396 (1997).

11 J. P. Perdew, K. Burke, and Y. Wang, Phys. Rev. B 54, 16533 (1996).

12 P. Nozières and D. Pines, Phys. Rev. 111, 442 (1958).

13 D. M. Ceperley and B. J. Alder, Phys. Rev. Lett. 45, 566 (1980).

14 W. E. Pickett and J. Q. Broughton, Phys. Rev. B 48, 14859 (1993).

15 G. Ortiz and P. Ballone, Phys. Rev. B 50, 1391 (1994); 56, 9970 (1997).

16 G. Ortiz, M. Harris, and P. Ballone, Phys. Rev. Lett. 82, 5317 (1999).

17 H. Yasuhara, Solid State Commun. 11, 1481 (1972).

18 A. W. Overhauser, Can. J. Phys. 73, 683 (1995).
19 K. Burke, J. P. Perdew, and M. Ernzerhof, J. Chem. Phys. 109, 3760 (1998).

20 D. J. W. Geldart, Can. J. Phys. 453139 (1967).

21 J. F. Dobson, J. Chem. Phys. 94, 4328 (1991); J. Phys.: Condens. Matt. 4, 7877 (1992).

22 M. W. C. Dharma-wardana and F. Perrot, Phys. Rev. Lett. 84, 959 (2000).

23 E. R. Davidson, Reduced Density Matrices in Quantum Chemistry (Academic Press, New York, 1976).

24 P. Ziesche, Phys. Lett. A 195, 213 (1994); Int. J. Quantum Chem. 60, 1361 (1996).

25 A. Gonis and T. C. Schulthess, J. Phys.: Condens. Matt. 10, 3535 (1998).

26 A. W. Overhauser, Ph.D. thesis, University of California, Berkley (1951); A. W. Overhauser, private communication.

27 P. Ziesche, J. Tao, M. Seidl, and J. P. Perdew, Int. J. Quantum Chem. 77, 819 (2000).

28 J. C. Kimball, Phys. Rev. A 7, 1648 (1973).

29 J. C. Kimball, Phys. Rev. B 14, 2371 (1976).

30 P. Ziesche, J. Mol. Struc. (Theochem) 527, 35 (2000).

31 See for example A.W. Overhauser and J.T. Tsai, Phys. Rev. B 13, 607 (1976).

32 D. C. Marinescu and J. J. Quinn, Phys. Rev. B 56, 1114 (1997).

33 M. Lein, E. K. U. Gross, and J. P. Perdew, Phys. Rev. B 61, 13431 (2000).

34 C. F. Richardson and N. W. Ashcroft, Phys. Rev. B 50, 8170 (1994).

35 D. Varsano, S. Moroni, and G. Senatore, Europhys. Lett. 53, 348 (2001).

36 M. Polini, G. Sica, B. Davoudi, and M.P. Tosi, J. Phys.: Condens. Matt. 13, 3591 (2001). This article appeared after ours was submitted. 